\title{
Microbial Performance of Food Safety Control and Assurance Activities in a Fresh Produce Processing Sector Measured Using a Microbial Assessment Scheme and Statistical Modeling
}

Njage, Patrick Murigu Kamau; Sawe, Chemutai Tonui; Onyango, Cecilia Moraa; Habib, I; Njagi, Edmund Njeru; Aerts, Marc; Molenberghs, Geert

Published in:

Journal of Food Protection

Link to article, DOI:

10.4315/0362-028X.JFP-16-233

Publication date:

2017

Document Version

Publisher's PDF, also known as Version of record

Link back to DTU Orbit

Citation (APA):

Njage, P. M. K., Sawe, C. T., Onyango, C. M., Habib, I., Njagi, E. N., Aerts, M., \& Molenberghs, G. (2017). Microbial Performance of Food Safety Control and Assurance Activities in a Fresh Produce Processing Sector Measured Using a Microbial Assessment Scheme and Statistical Modeling. Journal of Food Protection, 80(1), 177-188. https://doi.org/10.4315/0362-028X.JFP-16-233

\section{General rights}

Copyright and moral rights for the publications made accessible in the public portal are retained by the authors and/or other copyright owners and it is a condition of accessing publications that users recognise and abide by the legal requirements associated with these rights.

- Users may download and print one copy of any publication from the public portal for the purpose of private study or research.

- You may not further distribute the material or use it for any profit-making activity or commercial gain

- You may freely distribute the URL identifying the publication in the public portal 


\title{
Microbial Performance of Food Safety Control and Assurance Activities in a Fresh Produce Processing Sector Measured Using a Microbial Assessment Scheme and Statistical Modeling
}

\author{
PATRICK MURIGU KAMAU NJAGE, ${ }^{*}$ CHEMUTAI TONUI SAWE, ${ }^{1}$ CECILIA MORAA ONYANGO,${ }^{2}$ HABIB I, ${ }^{3}$ \\ EDMUND NJERU NJAGI, ${ }^{4,5}$ MARC AERTS, ${ }^{5}$ AND GEERT MOLENBERGHS ${ }^{5,6}$
}

\begin{abstract}
${ }^{1}$ Department of Food Science, Nutrition and Technology, and ${ }^{2}$ Department of Plant Science and Crop Protection, University of Nairobi, Nairobi, Kenya; ${ }^{3}$ College of Veterinary Medicine, Murdoch University, Murdoch 6150, Western Australia, Australia; ${ }^{4}$ Cancer Research UK Cancer Survival Group, London School of Hygiene \& Tropical Medicine, Keppel Street, London WCIE 7HT, UK; ${ }^{5}$ Interuniversity Institute for Biostatistics and Statistical Bioinformatics, Universiteit Hasselt, B-3590 Diepenbeek; and ${ }^{6}$ Interuniversity Institute for Biostatistics and Statistical Bioinformatics, Katholieke Universiteit Leuven,
\end{abstract} B-3000 Leuven, Belgium

MS 16-233: Received 27 May 2016/Accepted 8 September 2016/Published Online 22 December 2016

\begin{abstract}
Current approaches such as inspections, audits, and end product testing cannot detect the distribution and dynamics of microbial contamination. Despite the implementation of current food safety management systems, foodborne outbreaks linked to fresh produce continue to be reported. A microbial assessment scheme and statistical modeling were used to systematically assess the microbial performance of core control and assurance activities in five Kenyan fresh produce processing and export companies. Generalized linear mixed models and correlated random-effects joint models for multivariate clustered data followed by empirical Bayes estimates enabled the analysis of the probability of contamination across critical sampling locations (CSLs) and factories as a random effect. Salmonella spp. and Listeria monocytogenes were not detected in the final products. However, none of the processors attained the maximum safety level for environmental samples. Escherichia coli was detected in five of the six CSLs, including the final product. Among the processing-environment samples, the hand or glove swabs of personnel revealed a higher level of predicted contamination with E. coli, and $80 \%$ of the factories were E. coli positive at this CSL. End products showed higher predicted probabilities of having the lowest level of food safety compared with raw materials. The final products were $E$. coli positive despite the raw materials being E. coli negative for $60 \%$ of the processors. There was a higher probability of contamination with coliforms in water at the inlet than in the final rinse water. Four (80\%) of the five assessed processors had poor to unacceptable counts of Enterobacteriaceae on processing surfaces. Personnel-, equipment-, and product-related hygiene measures to improve the performance of preventive and intervention measures are recommended.
\end{abstract}

Key words: Correlated random effects joint models; Empirical Bayes estimates; Fresh produce; Generalized linear mixed models; Microbial assessment scheme

Among the food safety hazards, such as physical, chemical, and biological hazards, associated with foodborne illnesses linked to fresh and fresh-cut vegetables are microorganisms. Vegetables support the growth of microorganisms, including human pathogenic bacteria. These can be acquired from the production environment (soil, manure, and irrigation water) and handling during harvesting, processing (trimming, cutting, peeling, washing, and spinning), and packing $(21,52)$. In addition, cutting, slicing, and peeling during processing cause tissue damage, which releases nutrients and facilitates further microbial growth $(46,52)$. Given that fresh produce receives minimal or no preparation before consumption, contamination with patho-

\footnotetext{
* Author for correspondence. Present address: Division for Epidemiology and Microbial Genomics, National Food Institute, Technical University of Denmark, 2800 Kongens Lyngby, Denmark. Tel: +45358863 69; Fax: +45 358863 41; E-mail: kamau.patrick@gmail.com, panj@food.dtu.dk.
}

gens along the value chain can pose a serious risk to consumers. It is therefore critical to minimize initial contamination and bacterial growth, and to ensure the safety of fresh products $(21)$.

Producers and processors in the fresh and minimally processed fresh produce chain are required to design and implement effective food safety management systems (FSMS) according to the general principles of food hygiene of the Codex Alimentarius (6). The most commonly used FSMS standards and quality assurance guidelines include International Organization for Standardization (ISO) 22000:2005 (31) and the British Retail Consortium (BRC) (5) food safety standard (34). These FSMS standards and guidelines combine performance-based approaches such as inspection and sampling for testing. This is meant to evaluate the food safety control system and the performance of prerequisite programs such as good hygiene and sanitation programs (34). Integrated process-based ap- 
proaches such as FSMS, which combine both control and assurance activities, are also applied (43).

However, despite these interventions, bacterial pathogens, viruses, and pesticide residues remain a major concern, and foodborne outbreaks linked to fresh and minimally processed vegetables and fruits continue to be reported (50). This apparent ineffectiveness of applied FSMS in controlling food safety hazards has been attributed to differences in the interpretation and implementation of FSMS in the different sectors in the food chain, such as primary production, processing, and trade (36). The disparities in the interpretation and implementation of FSMS are influenced by technological development, resource availability, and access to information on standards (13). Situational elements that create risk in the decision-making processes and affect the design, implementation, and operation of FSMS also influence food safety output (48).

Consequently, stakeholders in the agrifood chain, such as consumers, sector organizations, regulatory agencies and food safety authorities, require information on the performance of FSMS. Such information enables the evaluation of the ability of implemented interventions to improve microbiological product safety (41). The most common method of FSMS evaluation entails checking compliance to specific requirements through steps such as the evaluation of records and documents, testing outcomes, and process control trends. However, this method does not provide any insight into FSMS performance, especially with respect to microbiological hazard levels. Several FSMS standards and guidelines such as ISO 22000 (31), BRC (5), and the Codex Alimentarius Hazard Analysis and Critical Control Point guidelines (6) recommend system audits and the evaluation of critical control points and prerequisite programs with microbial testing to confirm that the selected control measures are effective in eliminating or reducing microbial hazards to defined acceptable levels (35). But this system of verification may not give an indication of the level at which FSMS activities have been implemented in a companyspecific FSMS $(35,41,43)$. It also does not provide systematic information on the distribution of and variation in microbial contamination (34). A previous study (49) on the performance of FSMS control and assurance activities in view of contextual risk revealed weaknesses leading to the possibility of microbial contamination in the fresh produce FSMS. The fresh produce exporting companies work with initial materials characterized by a high risk of microbial contamination accompanied by partial physical intervention methods that are incapable of adequately reducing contamination levels (49). Such partially effective physical intervention methods include washing, sorting, grading, packaging, and chemical decontamination techniques whose aim is enhanced food safety through the reduction of physical contaminants and microbial load. Despite such risks, sampling for microbiological analysis is also variable, and some companies do not carry out microbial analyses (49).

A microbial assessment scheme (MAS) tool that allows the study of the actual microbial performance of core control and assurance activities in an implemented FSMS was developed by Jacxsens et al. (34). By tracking proximate indicators such as the levels of contamination before and after each control point, researchers can determine the impact of particular control measures (20). The MAS involves the analysis of selected microbial parameters in certain critical locations in a food establishment over a time interval, usually several months. Microbial safety level profiles are then assigned according to the extent to which criteria are met at the critical sampling locations (CSLs) (34). This indicates the food safety output of a FSMS and provides an overview of the microbial quality, hygiene, and safety levels of products and processes.

The MAS protocol has been successfully validated and used to highlight aspects requiring improvement in food processing establishments $(19,34,47,48)$. However, the MAS protocol lacks further inferential statistical treatment of the data, which will enable further conclusions to be drawn from the MAS results. It is therefore important to explore the potential of further data modeling to allow improved inference from the MAS.

Generalized linear mixed models (GLMMs) are used for modeling categorical data and accounting for clustering $(2$, $3,12,45,51)$. Correlated random-effects joint models, in contrast, are used for modeling multivariate clustered data (45). A detailed treatise on these models is provided in the online Supplemental Report (available at https://www. researchgate.net/publication/308397591_Microbial_ Performance_of_Food_Safety_Control_and_Assurance_ Activities_in_a_Fresh_Produce_Processing_Sector_ Measured_Using_a_Microbial_Assessment_Scheme_and_ Statistical_Modeling?ev=prf_pub).

The objective of this study was to assess the actual performance of microbiological control and assurance activities in an export fresh produce processing sector using the MAS protocol combined with further data modeling. The MAS protocol was followed by an analysis using GLMMs and correlated random-effects joint models. Our aim was to obtain insights on the effectiveness of the FSMS in preventing or reducing microbial contamination or hazards and to recommend improvements in fresh produce safety.

\section{MATERIALS AND METHODS}

Characterization of firms. The microbial assessment was carried out in five Kenyan fresh produce processing companies whose FSMS had earlier been studied (49) using an FSMS diagnostic instrument. The companies process various vegetables, including green beans, peas, leafy vegetables (spinach and pakchoi), spring onions, chives, broccoli, herbs, and stir-fry mixes (mixed vegetables), destined for export markets (see Table 1 for company characteristics of five processors). They obtain their produce mostly from their own farms and from subcontracted outgrowers with GlobalGAP certifications. We used the tool developed by Kirezieva et al. $(39,40)$ to study microbial contamination in primary production in order to further verify whether conditions at the supplying farms predisposed fresh produce to microbial contamination. This validated tool $(39,40)$ uses indicators and grids to rank the levels at which the indicators are implemented and to assess activities that are important for fresh produce and the system output in terms of microbiological and chemical food safety. The processing companies that we studied using the MAS were certified according to the BRC food safety management system standard, and some of the processors were 
TABLE 1. Characteristics of fresh produce processors assessed for microbial performance of safety management systems ${ }^{a}$

\begin{tabular}{|c|c|c|c|c|c|}
\hline \multirow[b]{2}{*}{ Characteristic } & \multicolumn{5}{|c|}{ Processor: } \\
\hline & P3 & P8 & P9 & P12 & P13 \\
\hline Total no. of employees & $50-249$ & $50-249$ & $50-249$ & $50-249$ & $50-249$ \\
\hline No. in QA department & 20 & 33 & 35 & 50 & 120 \\
\hline Products & $\mathrm{F}, \mathrm{V}$ & $\mathrm{V}, \mathrm{H}$ & $\mathrm{F}, \mathrm{V}$ & $\mathrm{V}$ & $\mathrm{V}, \mathrm{H}$ \\
\hline QA standard certified & $\mathrm{BRC}$ & $\mathrm{BRC}, \mathrm{CBS}$ & $\mathrm{BRC}, \mathrm{CBS}$ & $\mathrm{BRC}$ & $\mathrm{BRC}, \mathrm{CBS}$ \\
\hline Tonnage exported per annum & 3,000 & 7,800 & 5,000 & 7,000 & 7,000 \\
\hline
\end{tabular}

${ }^{a}$ QA, quality assurance; F, fruits; V, vegetables; H, herbs; BRC, British Retail Consortium; CBS, customer-based standards (Tesco Nature Source, Woolworths, or Marks and Spencer).

additionally certified to customer-based standards such as Tesco Nature Source, Woolworths, and Marks and Spencer. Operations, from sorting to packing, were mostly manual. Only processor P8 had a flume tank with a conveyor belt for produce washing operations; the rest had sets of wash tanks, after which the produce was transferred to spinning baskets for drying.

Food safety output assessment. A modified MAS protocol, as described by Jacxsens et al. (34), was used to determine the microbiological food safety output of the FSMS. The protocol involves the selection of (i) CSLs, (ii) microbiological parameters or indicators, (iii) sampling frequency, (iv) sampling and analytical methods, and (v) criteria for interpretation of the results $(34,48)$.

Selection of CSLs. A CSL is a location where contamination (or cross-contamination), growth, or survival of microorganisms can occur if the intervention or preventive strategy is not working effectively or where specific controls and corrective actions have to be carried out to achieve the desired output (34).

The product samples included the initial materials (CSL 1) and finished products or packaged vegetables (CSL 2). The food contact surface samples included swabs of the working tables or chopping boards, conveyer belts, spinning baskets, holding crates, and washing troughs (CSL 3), and swabs of the hand or glove of personnel (CSL 4). Both CSL 3 and CSL 4 are potential sources of cross-contamination and provide insights into the microbial performance of FSMS preventive measures. The quality of the washing water was also assessed. The use of water of poor microbial quality can lead to cross-contamination and an increase in the microbial load in the end product (19). Water samples were drawn at the inlet to holding tanks or washing troughs (CSL 5) and at the final rinse water trough (CSL 6). The incoming water was drawn from the inlet into the holding tanks from either a borehole or municipal lines. The final rinse water was sampled from the rinsing troughs or flume tanks after the addition of chlorine and before the introduction of the product. Our aim for this was to establish the microbial quality of the water used and the effectiveness of the added chlorine in controlling or eliminating the selected microbial indicators at CSL 6.

Selection of microbial parameters. Conditions at the growing location and the cultivation system affect the microbial safety of fresh produce (39). Escherichia coli, Salmonella spp., and Listeria monocytogenes were therefore selected as indicators of food safety. These microorganisms are indicative of preharvest contamination of vegetables from the production environment, human or animal sources, and inputs such as manure, irrigation water, and equipment $(21,37)$. E. coli and Enterobacteriaceae were analyzed as indicators of hygiene in the processing environment. Fresh produce safety is dependent on adequate hygiene and sanitation during processing (21). E. coli and Staphylococcus aureus were selected as indicators of personnel hygiene (1). Only personnel handling the final product or working in the packaging area were swabbed. Coliforms, E. coli, and enterococci, which are associated with fecal contamination (53), were selected as indicators of water quality.

Sampling frequency. Samples were drawn three times from each firm at different periods between October 2012 and June 2013. For each factory, two samples were collected for the initial product (CSL 1), final product (CSL 2), food contact surfaces (CSL 3 ), and hands and gloves (CSL 4) at the beginning and end of each working day. One sample each of the incoming water (CSL 5) and final rinse water (CSL 6) was also collected at the beginning and end of each working shift per factory. Therefore, 20 samples were collected per visit, and a total of 60 samples per company were analyzed, with a total of 300 samples analyzed.

Sampling and analytical methods. The sampling protocol and subsequent analysis of microbial parameters were performed using ISO methods. For destructive sampling of fresh-cut vegetables, we collected $250 \mathrm{~g}$ of vegetables in sterile stomacher bags. We sampled finished product samples from the packaged units. Nondestructive sampling of food contact surfaces and hands or gloves was performed by swabbing in accordance with ISO 18593:2004 (28) horizontal methods. A sterile steel template was used to delineate a sampling area. An area of $50 \mathrm{~cm}^{2}$ for the food contact surfaces and $25 \mathrm{~cm}^{2}$ for the hands or gloves of the personnel was swabbed using a sterile cotton swab premoistened in $10 \mathrm{ml}$ of sterile nutrient broth. All samples were stored and transported to the laboratory in a cool box at $\leq 4^{\circ} \mathrm{C}$. Sample preparation was done in accordance with ISO 6887-4:2003 (27). The test methods for microbial detection and enumeration are shown in Table 2. For enumeration and qualitative detection, a 25$\mathrm{g}$ sample of product was weighed in a stomacher bag and homogenized for $1 \mathrm{~min}$ in $225 \mathrm{ml}$ of buffered peptone water. The swab samples were vortexed for $10 \mathrm{~s}$, and the solution was incubated in the primary enrichment medium for the detection of pathogens or serially diluted for enumeration purposes.

ISO 21528-2:2004 (29) was used for Enterobacteriaceae enumeration, which involved a pour plate technique using violet red bile glucose agar. Colonies of presumptive Enterobacteriaceae were then subcultured onto nonselective medium and confirmed. The number was then calculated from the number of confirmed typical colonies per plate. For the detection and enumeration of $E$. coli, the method outlined ISO 7521:2005 (33) was used. This involved the inoculation of a test sample on violet red bile glucose agar plates followed by incubation at $37^{\circ} \mathrm{C}$ for $24 \pm 2 \mathrm{~h}$. Presumptive colonies were confirmed using Kovacs reagent (indole reaction). 

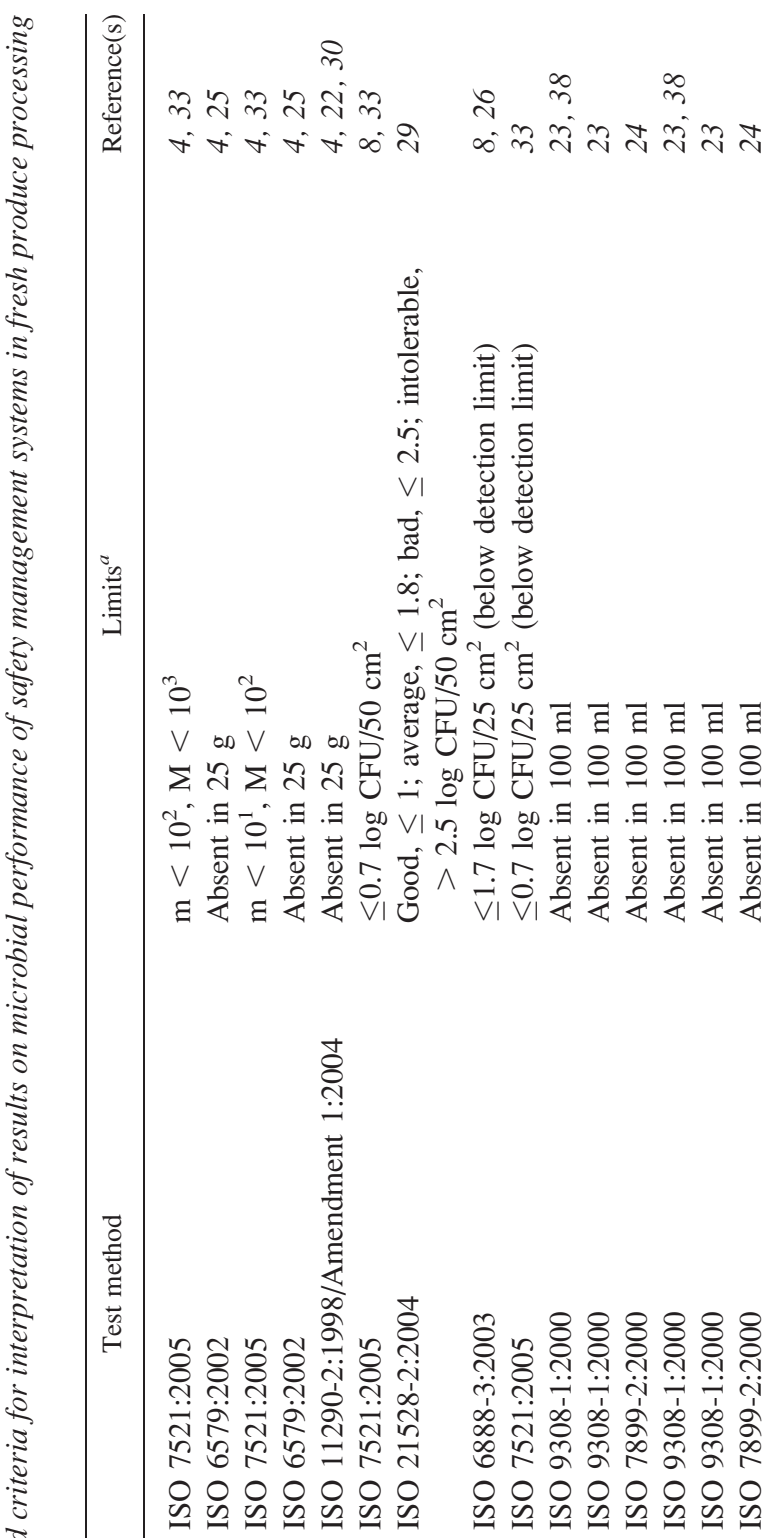

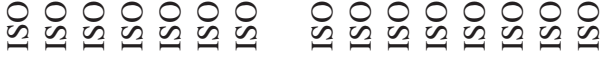$$
\text { ๖ }
$$

Microbiological criterion. The microbiological results for the product samples were interpreted using the criteria for readyto-eat vegetables given in European Commission Regulation 1441/ 2007 (4) and the International Commission on Microbiological Specifications for Foods (21). Microbiological guidelines established by the Laboratory of Food Microbiology and Food Preservation, Ghent University (8) were used to evaluate the food contact surfaces due to absence of legal criteria. Recommendations by Herbert et al. (18) were used to evaluate the hand swabs of personnel. The results for the water samples were interpreted using the requirements of the Kenya Standard potable water specification, KS 459-1:2007 (38). Table 2 gives a summary of the CSLs, analyzed parameters, test methods, and criteria for interpretation of the results.

Data analyses and interpretation of results from MAS. The MAS data were compiled and interpreted for compliance based on the criteria given in Table 2. A food safety level was attributed to each analyzed parameter on a scale of 1 to 3 . Level 3 represents a good safety performance in which legal criteria or guidelines are not exceeded; no improvement is required, and the current level of the FSMS is adequate to control the respective hazard. Level 2 indicates a moderate safety performance in which improvement is required for a specific control activity of the 


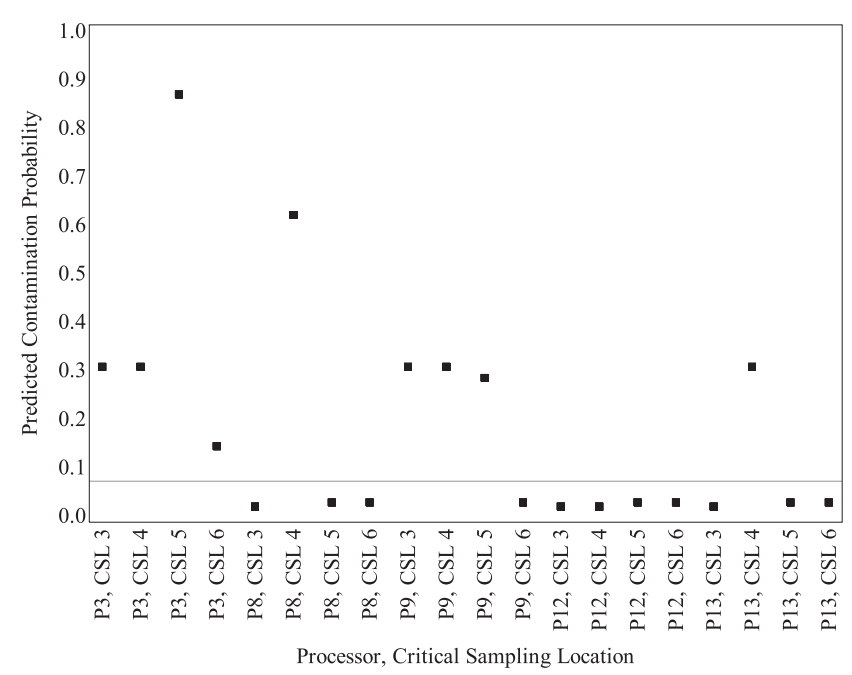

FIGURE 1. Predicted E. coli contamination probabilities in processor by CSL clusters for processing-environment samples, including food contact surface samples, hand or glove swabs of personnel, water at inlet, and at the final rinse water trough. Probabilities computed from estimates of a logistic regression model with random effects. Horizontal line represents the contamination probability in an average cluster, calculated by setting the random effect in the model to 0 (the mean of the random effects distribution).

FSMS. Level 1 represents a poor safety performance in which the legal criteria or guidelines are exceeded, and improvements are needed on several control activities in the FSMS (34). The sum of the food safety levels attributed to each microbial parameter per CSL is the microbial safety level profile (MSLP) score (34), where the maximum score per CSL was the number of microbial parameters multiplied by the highest performance level, level 3 . For instance, coliforms, enterococci, and E. coli were tested in CSL 6 , and therefore, the best or maximum score per processor for this CSL was 9. A score of 1 or 3 was attributed to the presence or absence, respectively, of a pathogen in a test sample. Table 2 gives the summary of the criteria for assigning the food safety levels.

The calculation of MSLPs enabled an overview of the FSMS output for each processor at specific CSLs. We then used Microsoft Office Excel 2007 (Microsoft, Redmond, WA) to construct bar graphs and scatter plots to visualize the MSLPs and variations in contamination among the companies across the CSLs.

Data analyses and interpretation of results by statistical modeling. We conducted statistical analyses to explore the differences in contamination levels across the CSLs and across the factories (processors). The statistical analyses was conducted on the data from E. coli, coliforms, and enterococci as representative of the tested indicators and because they were tested on at least two CSLs, making comparisons among CSLs possible. All the statistical analyses (exploratory data analyses and statistical modeling) were conducted using SAS software, version 9.4 (SAS Institute, Cary, NC).

To illustrate how the statistical models were implemented, sample SAS code with relevant annotation is provided in the online Supplemental Report, Appendix B. A number of different perspectives are possible, leading to a variety of statistical modeling approaches. For a detailed analysis of the modeling options available and reasons behind our choice of modeling approaches in this study, the reader is referred to the Supplemental Report.
Data analyses and interpretation of results by statistical modeling: the GLMM for processing-environment samples. For CSL 3, 4, 5, and 6, which are the processing-environment samples, we used GLMM, where the factory by CSL combinations were taken as random effects. Under this perspective, and using the empirical Bayes (EB) estimates, predictions of the probabilities of contamination in each factory by CSL combination was then made.

EB estimates $(2,3,45,51)$ are predictions of the random effects; random effects are useful not only in accounting for the cluster to cluster variability but also in constructing cluster-specific predictions. For $Y_{i j}$, the $j$ th sample in the $i$ th factory by CSL combination, $i=1, \ldots, 20$, the following GLMM was formulated:

$$
\begin{gathered}
\operatorname{logit}\left\{P\left(Y_{i j}=1 \mid b_{i}\right)\right\}=\beta_{0}+\beta_{i} \\
b_{i} \sim N\left(0, \sigma_{b}^{2}\right)
\end{gathered}
$$

where $\sigma_{b}^{2}$ is the variance of the random effects and $N$ is the normal distribution. The model assumes that the probability of contamination for a sample in any factory by CSL combination can be described by an overall, common parameter, $\beta_{0}$, and a factory by CSL combination-specific random effect, $b_{i}$.

The model was estimated using the NLMIXED procedure in SAS and using the Newton-Raphson optimization technique (45). The estimates of the two parameters of the model, the model intercept $\beta_{0}$ and the variance of the random effects $\sigma_{b}^{2}$, were -2.5526 and 5.6950, respectively, with a standard error of 0.7723 .

Using the model intercept and setting $b_{i}=0$, we computed the following quantity:

$$
\widehat{P}\left(Y_{i j}=1 \mid b_{i}=0\right)=\frac{e^{\widehat{\beta}_{0}}}{1+\widehat{e}^{\beta_{0}}}
$$

This quantity provides an estimate of the probability of contamination in factory by CSL combinations (clusters) that are at the mean of the random-effects distribution (the mean of the random-effects distribution being 0 ). This probability can be interpreted as the probability of contamination in an "average" cluster (i.e., factory by CSL combination). The estimated quantity was 0.0723 ; that is, the estimated probability of contamination in an "average" factory by CSL cluster was around $7 \%$.

Using the model intercept and the EB estimates of the random effects, we computed the predicted probability of contamination in each factory by CSL cluster:

$$
\widehat{P}\left(Y_{i j}=1 \mid b_{i}\right)=\frac{e^{\widehat{\beta_{0}}+\widehat{b_{i}}}}{1+\widehat{e}^{\widehat{\beta}_{0}+\widehat{b}_{i}}}
$$

where $\widehat{b}_{i}$ denotes the EB estimate for the cluster. The predictions are plotted in Figure 1 (the horizontal reference line depicts the probability of contamination in an average cluster).

Data analyses and interpretation of results by statistical modeling: $E$. coli in raw materials (CSL 1) and final products (CSL 2). We once again treated the factory by CSL combinations as clusters and followed a GLMM approach, but in this instance, we used the proportional odds model for the ordinal categorical outcome levels 1,2 , and 3 .

For these data, with $k$ representing the ordinal outcome level, the following GLMM was formulated for the $j$ th outcome in the $i$ th factory by CLS cluster $i, i=1, \ldots, 10$ :

$$
\begin{gathered}
\operatorname{logit}\left\{P\left(Y_{i j} \leq k \mid b_{i}\right)\right\}=\beta_{0 k}+b_{i} \quad k=1,2 \\
b_{i} \sim N\left(0, \sigma_{b}^{2}\right)
\end{gathered}
$$


The model assumes that the cumulative odds of lower levels of the outcome (i.e., lower levels of food safety) consist of the overall effects and a random effect of the cluster. The model was implemented using the NLMIXED procedure in SAS on the GLMM for processing-environment samples and using the Newton-Raphson optimization technique.

Based on the intercept parameter estimates, we computed the following three quantities:

$$
\begin{aligned}
& \widehat{P}\left(Y_{i j}=1 \mid b_{i}=0\right)=\frac{e^{\widehat{\beta}_{01}}}{1+\widehat{e}_{01}} \\
& \widehat{P}\left(Y_{i j}=2 \mid b_{i}=0\right)=\frac{\widehat{e}^{\widehat{\beta}_{02}}}{1+\widehat{e}_{02}}-\frac{\widehat{e}^{\beta_{01}}}{1+\widehat{e}^{\beta_{01}}} \\
& \widehat{P}\left(Y_{i j}=3 \mid b_{i}=0\right)=1-\frac{e^{\widehat{\beta}_{02}}}{1+\widehat{e}^{\beta_{02}}}
\end{aligned}
$$

These quantities represent the probabilities of each level of food safety in clusters that are the mean of the random-effects distribution (in average clusters). The computed probabilities are $28 \%$ for level 1, $17 \%$ for level 2, and $55 \%$ for level 3 .

Employing the EB estimates as before, we computed the predicted probability of each level of food safety in each factory by CSL combination:

$$
\begin{aligned}
& \widehat{P}\left(Y_{i j}=1 \mid b_{i}\right)=\frac{e^{\widehat{\beta}_{01}+\widehat{b}_{i}}}{1+\widehat{e}^{\widehat{\beta}_{01}+\widehat{b}_{i}}} \\
& \widehat{P}\left(Y_{i j}=2 \mid b_{i}\right)=\frac{e^{\widehat{\beta}_{02}+\widehat{b}_{i}}}{1+e^{\widehat{\beta}_{02}+\widehat{b}_{i}}}-\frac{e^{\widehat{\beta}_{01}+\widehat{b}_{i}}}{1+\widehat{e}^{\widehat{\beta}_{01}+\widehat{b}_{i}}} \\
& \widehat{P}\left(Y_{i j}=3 \mid b_{i}\right)=1-\frac{\widehat{e}^{\widehat{\beta}_{02}+\widehat{b}_{i}}}{1+\widehat{e}^{\widehat{\beta}_{02}+\widehat{b}_{i}}}
\end{aligned}
$$

The predicted probabilities of each level of food safety in each cluster are plotted separately in Figure $2 \mathrm{a}$ to $2 \mathrm{c}$ and plotted together in Figure 2d. Figure 2d facilitates a comparison of the probabilities of the different levels of food safety within each cluster.

Data analyses and interpretation of results by statistical modeling: coliforms and enterococci in water samples drawn at the inlet and final rinse water. The following correlated random-effects joint model was formulated for the $j$ th outcome from the $i$ th factory by CSL combination, $i=1, \ldots, 10$, where $Y_{1}$ represents the coliforms and $Y_{2}$ represents the enterococci.

$$
\begin{gathered}
\operatorname{logit}\left\{P\left(Y_{1 i j}=1 \mid b_{1 i}\right)\right\}=\beta_{10}+b_{1 i} \\
\operatorname{logit}\left\{P\left(Y_{2 i j}=1 \mid b_{2 i}\right)\right\}=\beta_{20}+b_{2 i} \\
b_{1 i}, b_{2 i} \sim N(0, D) \\
D=\left[\begin{array}{ll}
d_{11} & d_{12} \\
d_{21} & d_{22}
\end{array}\right]
\end{gathered}
$$

The model assumes that, for each of the two outcomes (coliforms and enterococci), the probability of contamination consists of an overall effect and a factory by CSL random effect. The random effect is outcome-specific (one random effect for coliforms and one for enterococci), with the two random effects being correlated. The parameters $d_{11}, d_{22}$, and $d_{12}=d_{21}$ are the variance of the random effects in the coliforms model, the variance of the random effects in the enterococci model, and the covariance between the two random effects, respectively. The model was implemented using the NLMIXED procedure in SAS on the GLMM for processing-environment samples and using the Newton-Raphson optimization technique.

The covariance parameter estimate is positive; a possible interpretation of the positive sign is that the higher the probability of coliform contamination in any factory by CSL cluster, the higher the probability of enterococci contamination in the cluster, and vice versa. However, a likelihood ratio test for the hypothesis that this covariance equals $0, H_{0}: d_{12}=0$; $H_{1}: d_{12} \neq 0$, results in $P=0.6547$. This means that there is no evidence that the covariance is different from 0 , and therefore, the positive covariance observed should not be overinterpreted or overemphasized.

We conducted the likelihood ratio test by comparing the log-likelihood of the correlated random effects joint model, $1_{1}=$ -49.4 , to the log-likelihood of a model imposing the restriction $d_{12}=0,11_{0}=-49.5$, with $1 \mathrm{df}$. The first model, in which the covariance is restricted to 0 , is equivalent to two separate GLMMs: one for coliforms and one for enterococci. Note that the two separate GLMMs can also be estimated as a "joint model," by simply setting $d_{12}=0$. When we refer to joint models from here on, we mean the correlated random-effects joint model, not the separate analyses estimated as a "joint model."

Given the nonsignificance of the covariance, we base further inferences on the two separate GLMMs, not on the joint model. Note that there are other theoretical and research objective-based considerations that still necessitate further inferences from the joint model. These include interest in hypotheses of a joint nature, missing data in one of the outcomes, and common fixed-effects parameters across the two outcomes (12); however, these considerations do not apply in our case.

Based on the intercept parameter estimates from the two GLMMs, we computed the probabilities of coliform and enterococci contamination in an average cluster as:

$$
\begin{gathered}
\widehat{P}\left(Y_{1 i j}=1 \mid b_{i}=0\right)=\frac{\widehat{e}^{\widehat{\beta}_{10}}}{1+\widehat{e}^{\hat{\beta}_{10}}} \\
\widehat{P}\left(Y_{2 i j}=1 \mid b_{i}=0\right)=\frac{\widehat{e}^{\widehat{\beta}_{20}}}{1+\widehat{e}^{\widehat{\beta}_{20}}}
\end{gathered}
$$

For coliforms, the probability of contamination was $0.0609 \%$, and for enterococci, it was $33.60 \%$.

Using the EB estimates, we computed the probabilities of coliform and enterococci contamination in the factory by CSL clusters as:

$$
\begin{gathered}
\widehat{P}\left(Y_{1 i j}=1 \mid b_{i}\right)=\frac{\widehat{e}^{\widehat{\beta}_{10}+\widehat{b}_{i}}}{1+\widehat{e}^{\widehat{\beta}_{10}+\widehat{b}_{i}}} \\
\widehat{P}\left(Y_{2 i j}=1 \mid b_{i}\right)=\frac{\widehat{e}^{\widehat{\beta}_{20}+\widehat{b}_{2 i}}}{1+\widehat{e}^{\widehat{\beta}_{20}+\hat{b}_{2 i}}}
\end{gathered}
$$

These probabilities are plotted in Figure 3 (as earlier, the horizontal lines depict the probabilities in average clusters). 
a

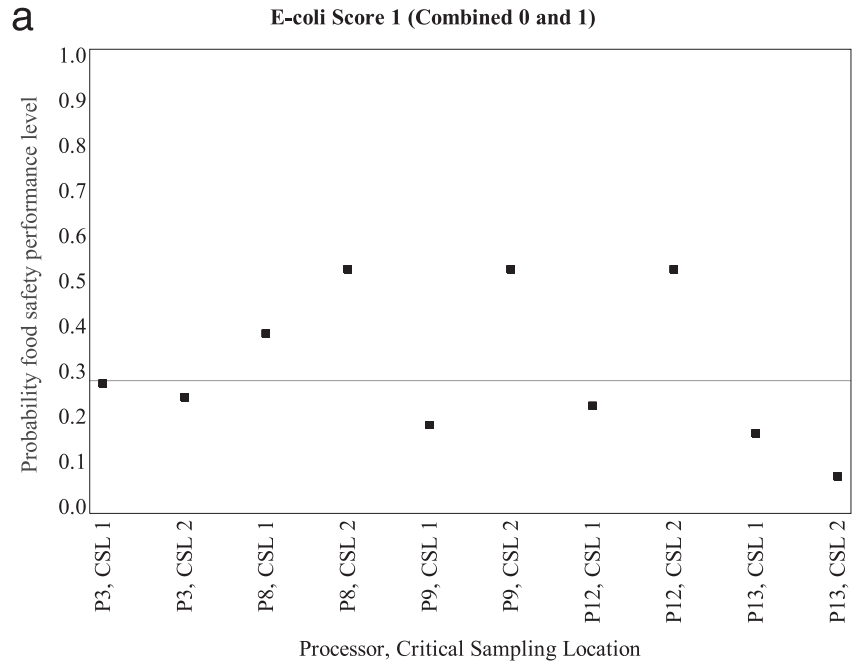

C

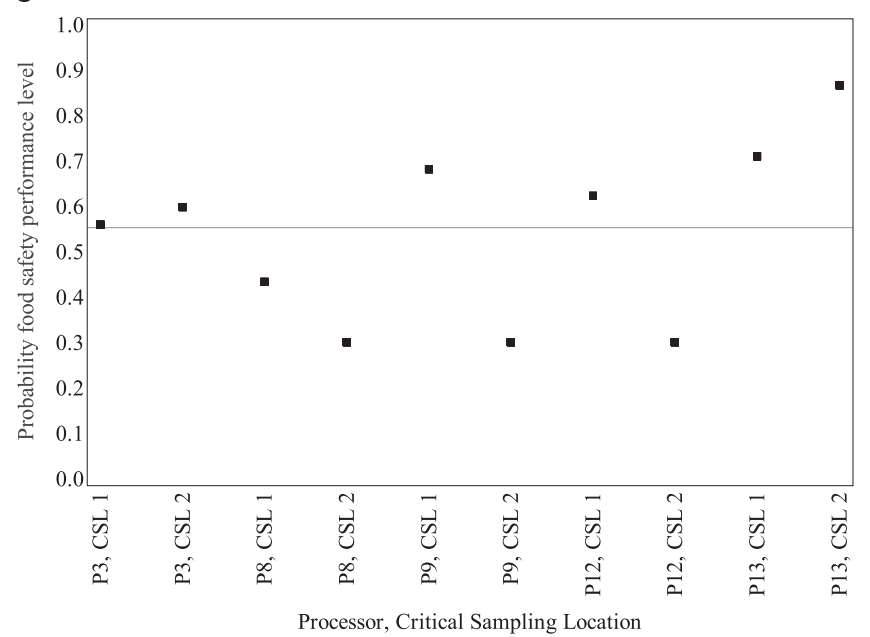

b

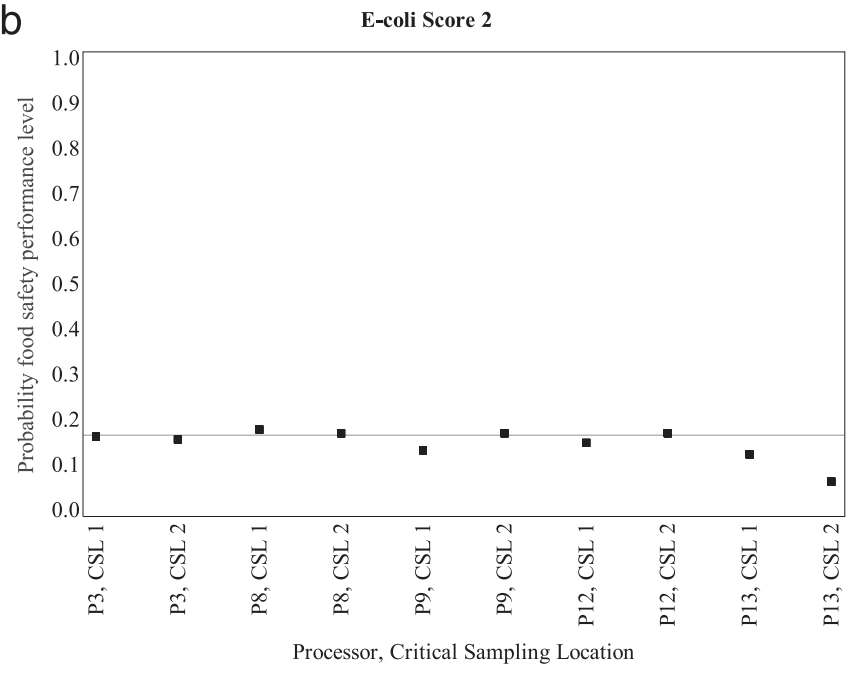

d

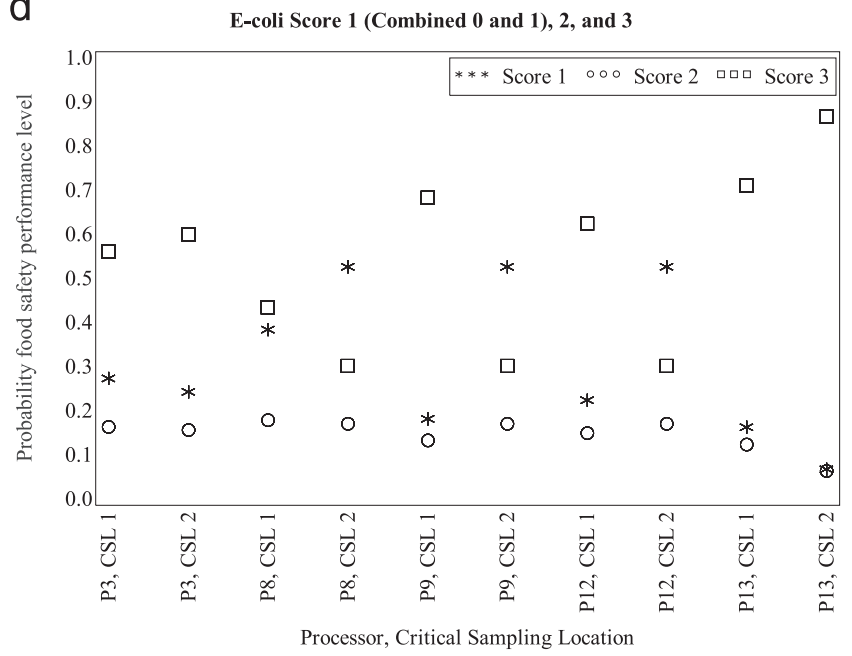

FIGURE 2. Probability of (a) lowest food safety performance level, level 1; (b) food safety performance level 2; (c) highest food safety performance level, level 3; and (d) each of the three food safety performance levels together in each processor by CSL combination for initial materials and final products. Horizontal reference lines depict the probability in an average cluster calculated by setting the random effect in the model to 0 (the mean of the random effects distribution). Level 1 represents a poor safety performance in which legal criteria or guidelines are exceeded and improvements are needed on several control activities in the FSMS. Level 3 represents a good safety performance, in which legal criteria or guidelines are not exceeded.

\section{RESULTS AND DISCUSSION}

Despite the certification of processors and exporters to standards such as the International Food Standard, GlobalGAP, and the BRC food safety standard, reports continue to appear about foodborne outbreaks due to contaminated fresh or minimally processed fruits and vegetables (46). An understanding of the critical factors influencing the performance of microbiological safety measures in fresh produce and horticultural production chains would ensure food safety in the short and long term. We illustrate here how the impact of established control measures can be assessed by using MAS and statistical modeling.

The results from the MAS are shown in Figure 4, and detailed results from exploratory analyses are shown in the online Supplemental Report, section 6. The indicator E. coli was detected in 66 samples from CSL 1 to CSL 5. E. coli therefore contributed most to the lower food safety levels. From statistical modeling in which we considered each CSL in each processor as a cluster, we found the probability of $E$. coli contamination in an average cluster to be around $7 \%$ in processing-environment samples, including food contact surface samples, hand or glove swabs of personnel, and incoming and final rinse water. Using the statistical model estimates, we computed the predicted probability of $E$. coli contamination in each cluster in the processing-environment samples (CSL 3, 4, 5, and 6); the predicted probabilities are plotted in Figure 1 (the horizontal reference line depicts the contamination probability in an average cluster). For all but one case, the predicted contamination probabilities in clusters involving the hands or gloves of personnel (CSL 4) were more than $7 \%$, with the lowest probability being $31 \%$ and the highest being $62 \%$. For the water samples at the final rinse water trough (CSL 6), in only one case was the contamination probability in a cluster more than $7 \%$. In the factories, the probabilities in all clusters involving processor P3 were more than $7 \%$, whereas no probability in any cluster 
a

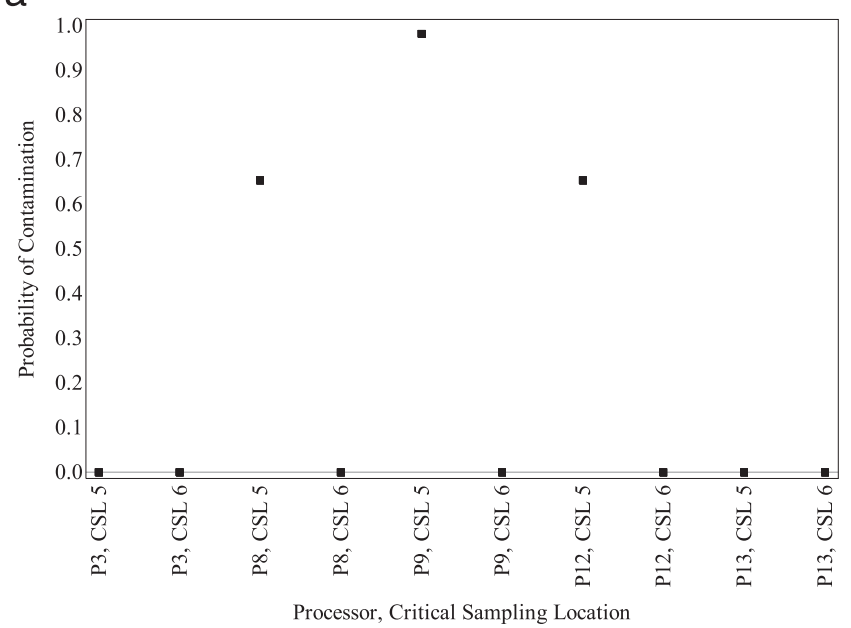

b

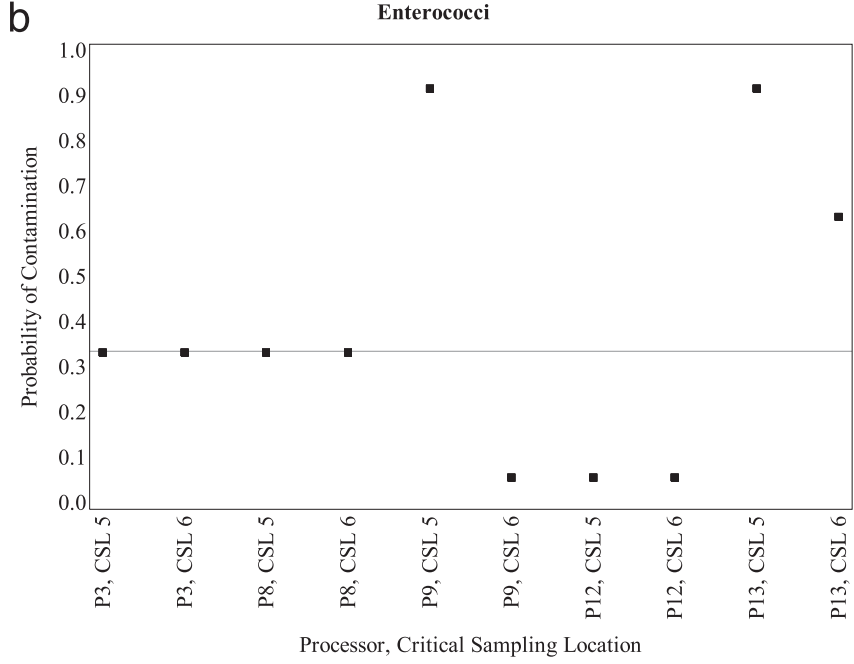

FIGURE 3. Probability of contamination of water at inlet and final rinse water with in each processor by CSL combination with (a) coliforms and (b) enterococci. Probabilities were computed from estimates of logistic regression models with random effects. Horizontal lines represent the probability in an average cluster, calculated by setting the random effect in the model to 0 (the mean of the random effects distribution).

involving P12 was more than 7\%. For P3, the proportion of E. coli-positive samples for any given CSL was at least $0.1667(16.67 \%)$, reaching a high of $100 \%$ in samples from the incoming water (CSL 5). This is in contrast to P12, where the proportion of E. coli-positive samples stood at $0 \%$ for all the CSLs. For processors P8, P9, and P13, the proportion of E. coli-positive samples was at least $33 \%$ for at least one of the CSLs. For the technical statistical details related to these results, as well all the other results of the statistical analysis discussed here, the interested reader is directed to the online Supplemental Report.
All the processors attained a food safety level of 3 for Salmonella spp. and L. monocytogenes. These pathogens were not detected. Nevertheless, some studies have recommended caution in interpreting results from the analysis of pathogens because they may occur at low prevalence levels in fresh produce $(<0.1$ to $1 \%)$ leading to low defect rates in food lots $(19,21)$.

Based on our statistical analyses, in which each CSL in each processor was again considered as a cluster, the probability of level 1 (the lowest level of food safety) was found to be $28 \%$, level 2 was $17 \%$, and level 3 (the highest
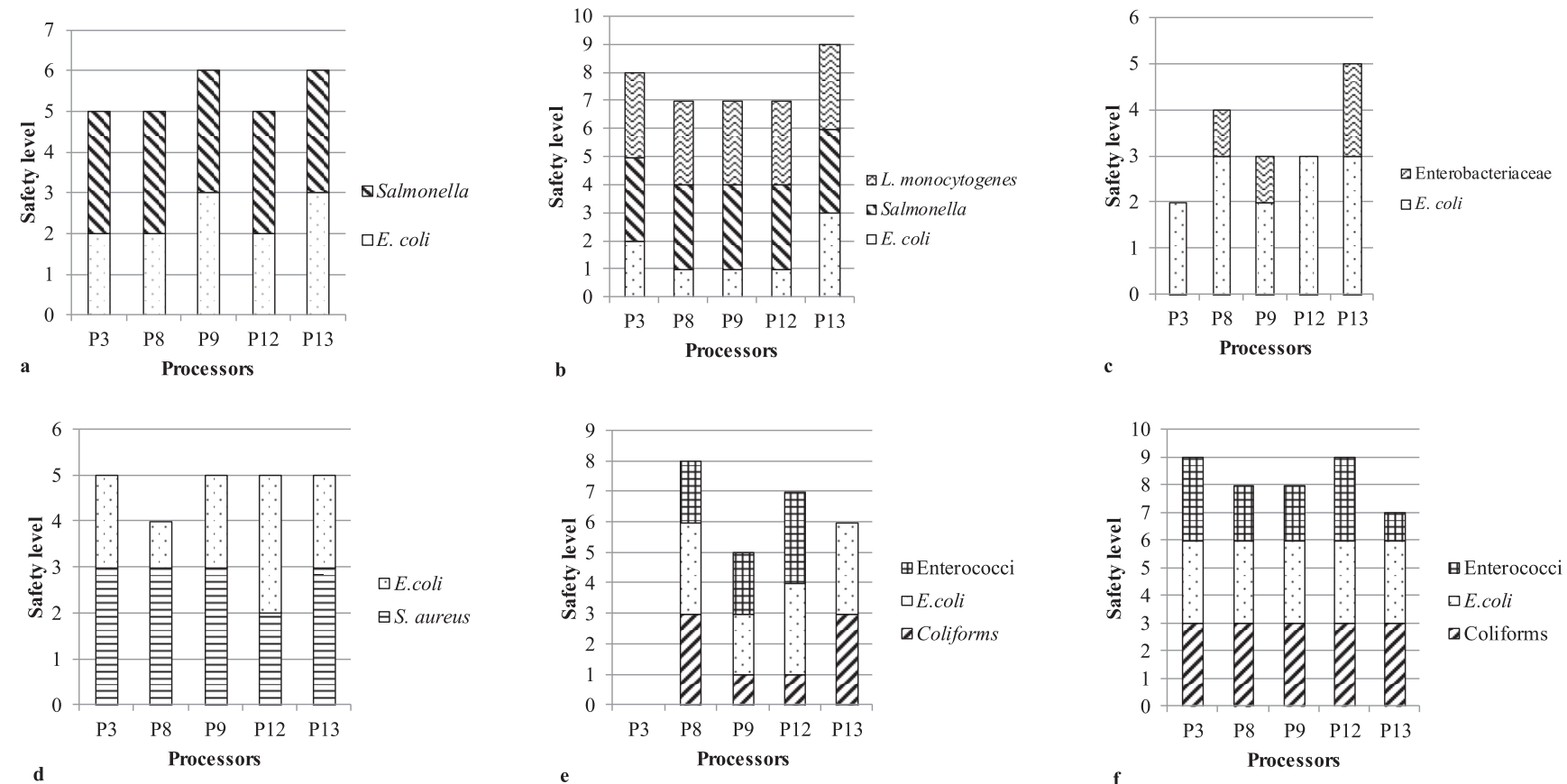

FIGURE 4. Microbial safety level profiles at CSLs for fresh produce processing firms (a) CSL 1, initial products; (b) CSL 2, final products; (c) CSL 3, product contact surfaces; (d) CSL 4, personnel hands or gloves; (e) CSL 5, incoming water; and (f) CSL 6, final rinse water. 
level) was $55 \%$ for the initial materials (CSL 1) and final products (packaged vegetables; CSL 2). Based on the model estimates, we also computed the predicted probability of each level of food safety in each cluster. These probabilities are plotted in Figure 2 (the horizontal reference line depicts the probability in average clusters). With regard to the lowest level of food safety, level 1, in only one cluster involving raw materials (CSL 1) was the estimated probability in an average cluster (28\%) exceeded, at $39 \%$ (Fig. 2a). For the second level of food safety, the probabilities in almost all clusters were very close to the probability in an average cluster (17\%; Fig. 2b). At the highest food safety performance level, level 3 , in four of the five clusters involving raw materials (CSL 1), the predicted probabilities were at or above the probability in an average cluster (Fig. 2c). An average food safety level of 2 was attributed from the MAS protocol for all the processors at CSL 1. These results indicate that preventive measures such as good agricultural practices, farm hygiene, and personnel hygiene can limit contamination (21). All the processors sourced their initial materials from GlobalGAP-certified farms with set minimum food safety assurance activity requirements. Such food safety assurance activities are a prerequisite for initial materials of good microbial quality (34). The verification of conditions at the GlobalGAPcertified supplying farms indicated that production conditions were not likely to pose a risk of microbial contamination of the raw materials. More rigorous FSMS controls are required to ensure that the microbial quality of the end product meets the food safety criteria whenever raw materials are contaminated (34). Poor performance at this level may put a strain on the FSMS controls at subsequent processing stages (41). The microbiological safety level of the initial materials therefore provides information on the potential safety risks associated with the raw materials, which in turn influences the rigorousness of FSMS interventions $(39,41)$.

A majority $(60 \%)$ of the processors performed poorly (were assigned food safety level of 1 ) with respect to $E$. coli in the final products, CSL 2 (Fig. 4b). A comparatively high number of samples were at the lowest food safety level for all processors except one at this CSL (see the Supplemental Report). In three of the five clusters involving final products, the probability of having the lowest food safety level was noticeably higher than in an average cluster (53\%). The poor performance is indicative of either inadequate decontamination processes or contamination from the processing environment, processing equipment, or human handling. The lack of efficiency of the sanitizer used in removing or killing pathogens on raw fruits and vegetables has also been attributed to structures and tissues that may harbor pathogens (14). The microbial quality of end products gives an indication of the effectiveness of the applied interventions in eliminating microbial hazards or reducing them to acceptable levels and of the overall performance of the FSMS (42). Such intervention measures include hygiene, sanitation, and decontamination processes (34). The most common method of decontamination among the processors was successive washing followed by rinsing with chilled (4 to $8^{\circ} \mathrm{C}$ ) chlorinated water (40 to $80 \mathrm{ppm}$ of chlorine) for an average of $5 \mathrm{~min}$. However, the $\mathrm{pH}$ and concentration of chlorine were not checked to ensure the maintenance of effective solutions for decontaminating the product. This can be attributed to a low level of company monitoring systems and to deficiencies in standards and tolerances with respect to product and process monitoring (49). For chlorine to be effective in decontamination, the combination of its concentration, $\mathrm{pH}$, and contact time with the product is important (52). Microbial cells present in the initial product might therefore persist in the end product if the active components and conditions necessary for their inactivation are not monitored. The risk posed by low level contamination can be enhanced by cross-contamination during washing, from surface moisture, and from temperature variation (7). This might explain the presence of E. coli in the final product even when it was not detected in the initial products from two processors (P9 and P12).

None of the processors achieved the maximum MSLP of 6 for CSL 3, product contact surfaces, which included produce holding crates, bowls, spinning baskets and their liners, conveyor belts, chopping boards, and work tables (Fig. 4c). The results for E. coli and Enterobacteriaceae at CSL 3 were variable over the sampling period. One processor (P3) had the lowest MSLP of 2 due to the presence of Enterobacteriaceae counts above the maximum limit. E. coli was detected in food contact surface swabs of two processors. These swabs were mainly from crates used to hold the product after spin drying and prior to packaging. The crates are made from plastic, and their construction may facilitate the adherence of microorganisms because they have perforations that may make cleaning difficult. Containers that come into contact with products should be designed and constructed in a way that makes them easy to clean, disinfect, and maintain to avoid contamination of the product (6). Of the processors we assessed, $80 \%$ had poor to unacceptable performance for the indicator Enterobacteriaceae (0 to $3.2 \log \mathrm{CFU} / \mathrm{cm}^{2}$ compared with the guidelines' acceptable maximum of $\log 2.5 \mathrm{CFU} / \mathrm{cm}^{2}$ ). This was despite the hygienic design of equipment and facilities being categorized as advanced in a previous study (49). Cleaning and sanitation procedures were therefore not effective in reducing microbial contamination to acceptable levels. Microorganisms may also adhere to food contact surfaces in form of biofilms, even after sanitation (14). The poor performance at CSL 3 can therefore compromise food safety through cross-contamination. The processors undertook the verification of their sanitation programs at intervals after cleaning and sanitation; however, the frequency of verification was not defined and therefore may not be satisfactory to ensure the effectiveness of the programs. Sanitation programs tailored and supported with appropriate instructions and verified as being effective in eliminating hazards should be implemented. These programs should be modified when the results of verification deviate from specifications $(6,41)$.

The highest proportion of $E$. coli-positive results was observed in the personnel hand swabs (CSL 4), with $33 \%$ of the samples being positive. MSLPs of 4 to 5 out of a maximum of 6 were recorded (Fig. 4d). S. aureus was detected in one personnel hand swab from one processor 
(P12) on one sampling occasion; hence, a food safety level of 2 was allocated. E. coli was detected in the hand swabs of $80 \%$ of the processors we assessed; hence, a food safety level of 1 or 2 was allocated for the indicator. The poor performance at CSL 4 with respect to $E$. coli might be the result of contact with an environment that contaminates hands with transient flora such as E. coli and Salmonella spp. (9). The CSL is a critical control point in the FSMS because most operations are manual and inadequate compliance with hygienic practices may compromise the safety of the end products. Personnel hygiene is important in the prevention of direct and indirect contamination of food because hands can contaminate food through skin-associated flora such as staphylococci $(1,9)$. However, no relationship was established between the detection of $E$. coli on the hands of personnel and in the final product. For example, although $E$. coli was not detected in any personnel swabs of $\mathrm{P} 12$, the indicator was detected in the final product on two occasions. This means that the E. coli contamination of end product may have originated from other sources in the processing environment. Both CSL 3 and 4 are potential sources of cross-contamination and provide insights into the microbial performance of FSMS preventive measures. Such preventive measures include the hygienic design of equipment and facilities, completeness of cleaning and sanitation, and compliance with hygiene requirements by personnel (35).

Water quality was assessed by testing enterococci and coliforms at both CSL 5 (incoming water) and CSL 6 (final rinse water), each with a maximum MSLP of 9. There were 30 samples that tested negative for coliforms that also tested negative for enterococci. Additionally, there were 8 samples that tested positive for coliforms that also tested positive for enterococci. The incoming water had the second highest level of E. coli contamination, with $27 \%$ samples testing positive. The percentage of positive samples for incoming water was $67 \%$ in two processors and $100 \%$ in one processor. Based on our statistical modeling results, in which each CSL in each processor was again considered as a cluster, the probabilities of coliforms and enterococci contamination in an average cluster were found to be 0.061 and $33.60 \%$, respectively. Using the model estimates, we also computed the contamination probabilities in the clusters, and these are plotted in Figure 3. For coliform contamination, in most clusters the probabilities were around the estimated probability in an average cluster $(0.061 \%)$, but the probabilities in three of the five clusters involving samples of the water at inlet were conspicuously much greater, at $65 \%$, with one cluster at $98 \%$. For enterococci contamination, the probabilities in most of the clusters were close to or below the probability in an average cluster $(33.60 \%)$, but in two clusters the predicted contamination probabilities for the samples of incoming water were more than $90 \%$ (Fig. 3b).

Washing is a partial intervention step in fresh produce processing; it is a critical step in reducing microbiological contamination and also removes some of the cell exudates that support microbial growth at cut surfaces $(6,17)$. Nevertheless, washing has been identified as a step through which microbial hazards can potentially be introduced, especially if the microbial quality of the water is unsatisfactory (19). Washing fresh-cut produce therefore requires the use of potable water to prevent the transfer of contamination from the water to the produce. Processor P3 had a food safety level of 0 for CSL 5 due to the detection of coliforms, E. coli, and enterococci in its incoming water (Fig. 4e). P9 also had a poor performance at CSL 5 due to the presence of coliforms and $E$. coli in the incoming water. P12 had an MSLP of 7 for CSL 5, and P8 attained an MSLP of 8. Enterococci were detected in all samples of the incoming water at P13; hence, an MSLP of 6 was allocated. This might be due to contamination or to the presence of biofilms in the piping system (16). Processors P3 and P13 sourced their water from boreholes, while the rest used municipal water. There was a better output at CSL 6 (Fig. 4f) because all the firms treated their water with chlorine prior to using it to rinse the product. In all clusters involving final rinse water, the predicted contamination probabilities were extremely small (only $0.045 \%$ ). Two processors attained the maximum MSLP of 9 at CSL 6. However, enterococci were still detected in four samples of final rinse water from three processors. The predicted contamination probability in one cluster reached $63 \%$ (Fig. 3). This may be due to either poor cleaning of the flume tanks or ineffective water treatment.

When the overall contamination profiles were considered, there was variation in E. coli contamination. This may be attributed to inadequate cleaning and sanitation as well as cross-contamination. Enterobacteriaceae counts at CSL 3 ranged from poor to unsatisfactory (food safety level of 1 or 2) in $80 \%$ of the processors. However, processor P13 had a better food safety ranking in most CSLs than did the other companies. Its final product met the criteria throughout the sampling period, and cleaning and sanitation were effective, with either the absence of $E$. coli contamination or a low variation when it was present. The processor's Enterobacteriaceae counts were also within the guidelines. E. coli was detected on only 1 of the 12 personnel swabs in P13. The FSMS control and assurance activities for P13 therefore seem effective in controlling microbial hazards, although the monitoring of water quality needs to be enhanced to ensure compliance with specifications.

Processor P3 had the lowest performing FSMS, as depicted by the MAS results (Fig. 4). Food safety levels at some CSLs for processor P3 indicated unsatisfactory performance of the control activities and prerequisite programs in preventing or reducing microbial hazards to acceptable levels. This poor output was mainly attributed to CSL 3 (food contact surfaces) and CSL 5 (incoming water). Equipment and facilities hygiene are therefore crucial in the prevention of cross-contamination throughout the processing environment. Practices such as insufficient washing of the wash tanks or flume tanks increase the potential of transferring $E$. coli contamination to the end product $(21,37)$.

The verification of cleaning and sanitation should therefore be improved by all the processors. The effectiveness of cleaning requires revalidation to improve general processing hygiene and reduce the possibility of cross-contamination. This will facilitate the development of more effective sanitation programs adapted for the various production zones to counter risk of cross-contamination. Cleaning and 
sanitation programs should be based on analyzed historical data for each company, and cleaning and sanitation should be tailored for specific equipment and facilities. In addition, the frequency of cleaning and sanitation should be based on the results of the verification activities.

Finally, from a statistical point of view, in the design of future studies it would be interesting to combine aspects of the MAS protocol with aspects of survey sampling methodology $(15,44)$. The MAS protocol could be used to define the CSLs, with survey sampling principles being used to specify a survey sampling design and to calculate the number of factories and the number of samples at each CSL in each factory that would be needed to achieve the objectives of the statistical analysis. A possibility in terms of the survey sampling design would be to consider the factories as clusters and the CSLs as strata; the appropriate calculations could then be conducted. Evidently, the intrafactory correlation would be an important input; this correlation could be estimated in the framework of a betabinomial model $(2,10,12,45)$.

In conclusion, we used a microbial assessment scheme together with statistical modeling to provide insights into the performance of microbial control and assurance activities in the fresh produce processing sector. The higher probabilities of coliform contamination in the inlet water compared with the final rinse water show the effectiveness of water treatment with chlorine prior to using the water in processing. In contrast, the higher probabilities of the presence of $E$. coli in the end product than in the raw materials indicate the ineffectiveness of the FSMS control measures, which may be due to inadequate monitoring at critical steps. The presence of $E$. coli in the final products when it was not detected in the initial product or on food contact surfaces indicates crosscontamination. Therefore, there is the possibility of spreading spot contamination during washing and because of poor cleaning and sanitation (preventive measures) of the flume tanks, inadequate intervention processes (decontamination), and inadequate monitoring systems.

Previous diagnostic results of FSMS activity indicate that $77 \%$ of the fresh produce processors operated at food safety levels ranging from basic to moderate (level 1 to level 2), which might be insufficient to address the risk of microbial contamination (49). The MAS results can therefore be related to the actual operation and efficacy of these measures in eliminating selected microbial hazards or reducing them to acceptable levels (34). Thus, better performance of control and assurance activities will be contingent on improvements in preventive measures, such as cleaning and sanitation programs, personnel hygiene, and hygienic design of equipment and facilities, that have been tailored for fresh produce. The reevaluation of intervention measures and adequate monitoring methods are also necessary to assure food safety. We suggest a future research direction in which the MAS protocol is used to define the CSLs, and survey sampling principles are used to specify a survey sampling design and to calculate the number of factories and the number of samples at each CSL in each factory needed to achieve the specified statistical analysis objectives.

\section{ACKNOWLEDGMENTS}

We acknowledge the fresh produce processors for making this study possible by opening their premises and systems to facilitate sampling for microbial assessment. We also acknowledge the training from EU Veg-iTrade project partners, especially at Ghent University, Belgium, through which the microbial assessment tool was previously developed and validated.

\section{REFERENCES}

1. Aarnisalo, K., K. Tallavaara, G. Wirtanen, R. Maijala, and L. Raaska. 2006. The hygienic working practices of maintenance, personnel and equipment hygiene in the Finnish food industry. Food Control 17:1001-1101.

2. Aerts, M., H. Geys, G. Molenberghs, and L. M. Ryan. 2002. Topics in modelling of clustered data. Chapman \& Hall, Boca Raton, FL.

3. Agresti, A. 2002. Categorical data analysis, 2nd ed. John Wiley \& Sons, New York.

4. Anonymous. 2007. Commission Regulation (EC) No 1441/2007 of 5 December 2007 amending Regulation (EC) No 2073/2005 on microbiological criteria for foodstuffs. Off. J. Eur. Union L 322:1229.

5. British Retail Consortium (BRC). 2011. BRC global standard for food safety, issue 6. Stationery Office, London.

6. Codex Alimentarius Commission (CAC). 2003. Hazard analysis and critical control point (HACCP) system and guidelines for its application. ANNEX to recommended international code of practice/general principles of food hygiene. CAC/RCP 1-1969, Rev 4. Food and Agriculture Organization of the United Nations and World Health Organization, New York.

7. Danyluk, M. D., and D. W. Schaffner. 2011. Quantitative assessment of the microbial risk of leafy greens from farm to consumption: preliminary framework, data, and risk estimates. J. Food Prot. 74:700-708.

8. Debevere, J. M., M. Uyttendaelle, F. Devlieghere, and L. Jacxsens. 2006. Microbiological guide values and legal microbiological criteria. Laboratory of Food Microbiology and Food Preservation, Ghent University, Ghent, Belgium.

9. Dijk, R., D. van den Berg, R. R. Beumer, E. de Boer, A. Dijkstra, P. Kalkmand, H. Stegeman, M. Uyttendaelle, and H. Veenendaal. 2007. Microbiologie van Voedingsmiddelen: methods, principles en criteria (vierdedruk). UitgeverijKeesing Noordervliet, Houten, The Netherlands.

10. Faes, C., G. Molenberghs, M. Aerts, G. Verbeke, and M. G. Kenward. 2009. The effective sample size and an alternative small-sample degrees-of-freedom method. Am. Stat. 63:389-399.

11. Fieuws, S., and G. Verbeke. 2004. Joint modelling of multivariate longitudinal profiles: pitfalls of the random-effects approach. Stat. Med. 23:3093-3104.

12. Fitzmaurice, G., M. Davidian, G. Verbeke, and G. Molenberghs. 2009. Longitudinal data analysis. Chapman \& Hall, Boca Raton, FL.

13. Food and Agriculture Organization of the United Nations. 2007. Bridging the gap between food safety policies and implementation. C2007/INF/19. Thirty-fourth session, 17 to 24 November 2007, Rome.

14. Frank, J. E. 2001. Microbial attachment to food and food contact surfaces. Adv. Food Nutr. Res. 43:319-370.

15. Groves, R. M., F. J. Fowler, Jr., M. P. Couper, J. M. Lepkowski, E. Singer, and R. Tourangeau. 2004. Survey methodology. John Wiley \& Sons, New York.

16. Hallam, N. B., J. R. West, C. F. Forster, and J. Simms. 2001. The potential for biofilm growth in water distribution systems. Water Res. 35:4063-4071.

17. Harris, L. J., J. N. F. Farber, L. R. Beuchat, M. E. Parish, T. V. Suslow, E. H. Garrett, and F. F. Busta. 2003. Outbreaks associated with fresh produce: incidence, growth and survival of pathogens in fresh and fresh-cut produce. Compr. Rev. Food Sci. Food Saf. 2:78141.

18. Herbert, M., T. Donovan, and P. Manger. 1990. A study of the microbial contamination of working surfaces in a variety of food premises using the traditional swabbing technique and commercial contact slides. Public Health Laboratory Service, Ashford, UK. 
19. Holvoet, K., L. Jacxsens, I. Sampers, and M. Uyttendaelle. 2012. Insight into the prevalence and distribution of microbial contamination to evaluate water management in the fresh produce processing industry. J. Food Prot. 75:671-681.

20. International Commission on Microbiological Specifications for Foods. 2006. Microorganisms in foods. Use of epidemiological data to measure the impact of food safety control programs. Food Control $17: 825-837$.

21. International Commission on Microbiological Specifications for Foods. 2011. Microorganisms in foods, 8: Use of data for assessing process control and product acceptance. Springer Science, New York.

22. International Organization for Standardization. 1998. Microbiology of food and animal feeding stuffs - horizontal method for the detection and enumeration of Listeria monocytogenes - part 2: Enumeration method. ISO 11290-2. International Organization for Standardization, Geneva.

23. International Organization for Standardization. 2000. Water qualitydetection and enumeration of Escherichia coli and coliform bacteria-part 1: Membrane filtration method. ISO 9308-1. International Organization for Standardization, Geneva.

24. International Organization for Standardization. 2000. Water qualitydetection and enumeration of intestinal Enterococci-part 2: Membrane filtration method. ISO 7899-2. International Organization for Standardization, Geneva.

25. International Organization for Standardization. 2002. Microbiology of food and animal feeding stuffs - horizontal method for the detection of Salmonella spp. ISO 6579. International Organization for Standardization, Geneva.

26. International Organization for Standardization. 2003. Microbiology of food and animal feeding stuffs-horizontal method for the enumeration of coagulase-positive Staphylococci (Staphylococcus aureus and other species)—part 3: Detection and MPN technique for low numbers. ISO 6888-3. International Organization for Standardization, Geneva.

27. International Organization for Standardization. 2003. Microbiology of food and animal feeding stuffs_-preparation of test samples, initial suspension and decimal dilutions for microbiological examinationpart 4: Specific rules for the preparation of products other than milk and milk products, meat and meat products, and fish and fishery products. ISO 6887-4. International Organization for Standardization, Geneva.

28. International Organization for Standardization. 2004. Microbiology of food and animal feeding stuffs-horizontal methods for sampling techniques from surfaces using contact plates and swabs. ISO 18593. International Organization for Standardization, Geneva

29. International Organization for Standardization. 2004. Microbiology of food and animal feeding stuffs - horizontal methods for the detection and enumeration of Enterobacteriaceae-part 2: Colony-count method. ISO 21528-2. International Organization for Standardization, Geneva.

30. International Organization for Standardization. 2004. Modification of the enumeration medium. ISO 11290-2:1998/Amd 1. International Organization for Standardization, Geneva.

31. International Organization for Standardization. 2005. Food safety management systems-requirements for any organization in the food chain. ISO 22000. International Organization for Standardization, Geneva.

32. International Organization for Standardization. 2005. General requirements for the competence of testing and calibration laboratories. ISO/IEC 17025. International Organization for Standardization, Geneva.

33. International Organization for Standardization. 2005. Microbiology of food and animal feeding stuffs - horizontal method for the detection and enumeration of presumptive $E$. coli-most probable number technique. ISO 7521. International Organization for Standardization, Geneva.

34. Jacxsens, L., J. Kussaga, P. A. Luning, M. Van de Spiegel, F. Devlighere, and M. Uyttendaele. 2009. A microbial assessment scheme to support microbial performance measurements of food safety management systems. Int. J. Food Microbiol. 134:113-125.

35. Jacxsens, L., M. Uyttendaelle, F. Devlieghere, J. Rovira, S. Osés Gomez, and P. A. Luning. 2010. Food safety performance indicators to benchmark food safety output of a food safety management system. Int. J. Food Microbiol. 141:180-187.

36. Jin, S. S., J. Zhou, and J. Ye. 2008. Adoption of HACCP system in the Chinese food industry: a comparative analysis. Food Control 19:823-828.

37. Johnston, L., L. Jaykus, D. Moll, M. C. Martinez, J. Anciso, B. Mora, and C. L. Moe. 2005. A field study of the microbiological quality of fresh produce. J. Food Prot. 68:1840-1847.

38. Kenya Bureau of Standards. 2007. Kenya Standard KS 459-1:2007: Drinking water, specification part 1: The requirements for drinking water. Nairobi, Kenya.

39. Kirezieva, K., L. Jacxsens, M. Uyttendaele, M. A. J. S. Van Boekel, and P. A. Luning. 2013. Assessment of food safety management systems in the global fresh produce chain. Food Res. Int. 52:230-242.

40. Kirezieva, K., J. Nanyunja, L. Jacxsens, J. G. A. J. van der Vorst, M. Uyttendaele, and P. A. Luning. 2013. Context factors affecting design and operation of food safety management systems in the fresh produce chain. Trends Food Sci. Technol. 32:108-127.

41. Luning, P. A., L. Bango, J. Kussaga, J. Rovira, and W. J. Marcelis. 2008. Comprehensive analysis and differentiated assessment of food safety control systems: a diagnostic instrument. Trends Food Sci. Technol. 19:522-534.

42. Luning, P. A., and W. J. Marcelis. 2007. A conceptual model of food quality management functions based on a techno-managerial approach. Trends Food Sci. Technol. 18:159-166.

43. Luning, P. A. and W. J. Marcelis. 2009. A food quality management research methodology integrating technological and managerial theories. Trends Food Sci. Technol. 20:34-44.

44. Milanzi, E., E. N. Njagi, L. Bruckers, and G. Molenberghs. 2015. Data representativeness: issues and solutions. EFSA Supporting Publications 12(2). doi:10.2903/sp.efsa.2015.EN-759.

45. Molenberghs, G., and G. Verbeke. 2005. Models for discrete longitudinal data. Springer, New York.

46. Olaimat, N. A., and R. A. Holley. 2012. Factors influencing the microbial safety of fresh produce: a review. Food Microbiol. 32:1-19.

47. Osés, S. M., P. A. Luning, L. Jacxsens, S. Santillana, I. Jaime, and J. Rovira. 2012. Microbial performance of food safety management systems implemented in the lamb production chain. J. Food Prot 75:95-103.

48. Sampers, I., L. Jacxsens, P. A. Luning, W. J. Marcelis, A. Dumoulin, and M. Uyttendaelle. 2010. Performance of food safety management system in poultry meat processing plants in relation to Campylobacter spp. contamination. J. Food Prot. 73:1447-1457.

49. Sawe, C. T., C. M. Onyango, and P. M. K. Njage. 2014. Current food safety management systems in fresh produce exporting industry are associated with lower performance due to context riskiness: case study. Food Control 40:335-343.

50. Van Boxstael, S., I. Habib, L. Jacxsens, M. De Vocht, L. Baert, E. Van de Perre, A. Rajkovic, F. Lopez-Galvez, I. Sampers, P. Spanoghe, B. De Meulanaer, and M. Uyttendaelle. 2013. Food safety issues in fresh produce: bacterial pathogens, viruses and pesticide residues indicated as major concerns by stakeholders in the fresh produce chain. Food Control 32:190-197.

51. Verbeke, G., and G. Molenberghs. 2000. Linear mixed models for longitudinal data. Springer, New York.

52. World Health Organization. 1998. Food safety issues: surface decontamination of fruits and vegetables eaten raw: a review. WHO/FSF/FOS/98.2. Available at: http://www.who.int/foodsafety/ publications/. Accessed December 2014.

53. World Health Organization. 2006. Guidelines for drinking water quality, 3rd ed. World Health Organization, New York. 